\title{
Estado Documental de la Evaluación de la Huella Hídrica
}

\section{Documentary Status of the Water Footprint Assessment}

DOI: https://doi.org/10.17981/ijmsor.04.01.06

Research Article - Reception Date: Apr 24, 2019- Acceptance Date: Jul 27, 2019

Regino R. Alvarez Cancio-Bello

Universidad “Carlos R. Rodríguez”. Cienfuegos (Cuba)

ralvarez@ucfedu.cu

To reference this paper:

R. Alvarez Cancio-Bello "Estado documental de la evaluación de la huella hídrica", IJMSOR, vol. 4, no. 1, 2019. https://doi. org/10.17981/ ijmsor.04.01.06

Resumen-- El trabajo trata las metodologías y los indicadores para la evaluación de los impactos de uso del agua dulce, los métodos existentes analizan fundamentalmente la cantidad de agua usada con relación a los impactos ocasionados. Teniendo en cuenta que hay una necesidad reconocida para considerar los impactos, específicamente sobre bases del ciclo de vida, la problemática es que se consideran insuficientes los datos o no son confiables en las bases de datos del ciclo de vida del uso del agua, además no coincide el método de valoración del impacto del ciclo de vida para lo relacionado con los impactos estimados en el uso del agua dulce, se resaltan estas dificultades y se analizan las ventajas, limitaciones, diferencias en los resultados entre diversos métodos y se observa la necesidad de una metodología perfeccionada para la evaluación de los impactos del uso del agua sobre bases del ciclo de vida.

Palabras clave: Métodos; huella hídrica; agua; indicadores; impactos; ciclo de vida.

\begin{abstract}
This work deals with the methodologies and indicators for the evaluation of the blows of use of fresh water, the analysis methods fundamentally analyze the amount of water used in relation to the blows caused. Taking into account that there is a recognized need to consider impacts, specifically on a life cycle basis, the problem is that the data is considered insufficient or unreliable in the life cycle databases of water use, in addition, it is not coincide with the method of assessing the impact of the life cycle for what is related to the estimated impacts on the use of fresh water, highlight these difficulties and analyze the advantages, limitations, differences in the results between various methods and observe the need for a methodology perfected for evaluating the impacts of water use on the basis of the life cycle.
\end{abstract}

Keywords: Methods; water footprint; water; indicators; impacts; lifecycle. 


\section{Desarrollo}

\section{A. $L C I$}

Los métodos fundamentales utilizados para la cuantificación del uso del agua en el inventario del ciclo de vida (LCI) son los siguientes:

\section{1) El método de Hoekstra}

Está sustentado en el concepto de agua virtual [1]. Se introdujo el concepto de huella hídrica el cual ha sido posteriormente desarrollado y perfeccionado como un método para cuantificar el uso del agua para un producto, servicio o nación [2], [3].

La huella hídrica constituye la suma de toda el agua usada en una serie de suministro, comprimiendo agua azul, verde y gris. El agua azul declarada como el volumen de agua dulce extraída desde ríos, sacada y llevada por conductoras de agua, la cantidad de agua de lluvia usada por las plantas es referida como agua verde y el agua gris es la provocada por el impacto de la contaminación de los recursos de agua que representa el volumen de agua fresca necesaria para diluir la contaminación de manera que permanezca la calidad del agua que queda sobre los estándares de calidad de agua establecidos por las regulaciones. Este criterio ha sido usado calculando la huella hídrica de varios productos de la agricultura, por ejemplo:

La huella hídrica del vino ha sido calculada en 120 1/vas, del bioetanol (del maíz) en $110^{3}$ ) y de la carne de vaca en" $500 \mathrm{l} / \mathrm{kg}$, lo cual es concebido para ser usado como una herramienta para el desarrollo de estrategias de reducción del agua social [4] y para la rotulación de la huella hídrica del agua de productos [5], aunque estos criterios pudieran proporcionar resultados falseados [4], [6]. La cuantificación del uso del agua es polémica debido a la inclusión del agua verde, lo cual no provoca que se afecte la disponibilidad de agua azul la que pudiera no ser considerada; además, la extracción de agua preferiblemente de consumo es con frecuencia usada en la valoración de la huella hídrica del agua azul. Esto pudiera ser una dificultad, especialmente en el caso del uso del agua industrial cuando solo una parte pequeña del agua extraída es consumida (evaporada en torres de enfriamiento o incluida en el producto) y el residuo es con frecuencia descargado a un estanque de agua (agua de enfriamiento o efluentes industriales de agua residual).

Con relación a la huella hídrica del agua gris es debatido que los impactos ambientales del agua gris son más convenientes dirigirlos en otras categorías de impactos tal como toxicidad [7]. También en ausencia de un método para la cuantificación de los volúmenes de dilución para su asimilación, la estimación de la huella hídrica del agua gris es subjetiva.

\section{2) El método de Milá Canals}

El método basa el uso del agua en el nivel de una cuenca de río. Por lo tanto, para este método, la fuente de agua y el tipo de uso del agua dulce pueden ser incluidos en el inventario del ciclo de vida [7]. Con respecto a la fuente, este sigue clasificando las fuentes de agua como agua azul y verde. La categoría de agua azul está diferenciada adicional en 3 tipos: Flujo de rio o lago, fondo acuífero y reserva fósil; y el agua de uso es repartida en 2 categorías: de uso evaporativo y no evaporativo.

Finalmente es definida como agua de retorno la fuente de agua dulce después de su uso y disponible para uso adicional. Está propuesto valorar la disponibilidad del agua de lluvia sobre los efectos en el uso de la tierra que tiene en cuenta cambios en el sistema de producción, relativo a la referencia en el uso de la tierra.

\section{3) El método de Pfister}

El método fundamenta el uso del agua sobre una escala pequeña respecto al método de Milá Canals, tomando la cuenca como área del centro. Á diferencia de lo que abordan los métodos anteriores, este método considera solo el agua azul. Este método diferencia 2 categorías de uso de agua: El agua de consumo y el agua de calidad degradada o residual. La principal diferencia entre el método de Milá Canals y este método que se trata es que el agua descargada a alguna cuenca es tratada aquí como consumida, mientras el método de Milá Canals aborda consideraciones del agua descargada a una fuente de agua dulce como un uso no evaporativo. A diferencia de lo que aborda Milá Canals, este método recomienda que la descarga del agua residual debe ser valorada por la pérdida de la calidad del agua, no haciendo referencia sobre como esto pudiera ser ejecutado.

\section{B. Valoración del impacto en el inventario del ciclo de vida. Métodos para la valoración de los impactos del uso del agua}

Los cambios en la disponibilidad de agua dulce debido al consumo de agua y la degradación de la calidad causada por la descarga de agua residual puede tener impactos significantes sobre el ecosistema y la salud humana, actualmente los métodos de valoración del inventario del ciclo de vida no consideran los impactos del uso del agua [7], [8].

\section{1) El método de eco-escasez}

El método se basa en el principio de objeto a distancia, proporcionando eco-factores para varios impactos medioambientales incluyendo el uso de agua. Aquí el uso del agua es definido como la entrada total de agua dulce extraída para la producción o consumo. 
Para calcular los impactos de escasez de agua, a cada categoría entonces le es asignada un factor eco basado en el valor promedio de disponibilidad para consumo de agua [9]. El eco-factor es calculado por la ecuación siguiente:

Eco-factor $=\frac{1 x E P}{F n} *(W T A)^{2}\left(\frac{1}{2096}\right)^{2} * C \quad\left[E P / m^{3}\right]$

Fn: Factor de normalización para el consumo de agua.

WTA: Rango de escasez o rango de uso de agua de los recursos disponibles.

20\%: Porcentaje de demanda tolerable sobre recursos de agua (20\% acordado por la OECD, 2004)

$C$ : $\quad$ Constante (10\%) a) para obtener valores numéricos presentables.

Este método puede ser aplicado en la región o país con nivel de cuenca. Sin embargo, los eco-factores nacionales proporcionan una limitada percepción local por dentro para países heterogéneos tal como EU, China, Australia y la India. El uso del método WTA basado en los datos anuales resulta que no se obtengan las variaciones de los datos estacionales, además el uso del WTA puede sobrestimar el estrés hídrico, son asignados factores de carga muy altos para países en la categoría extrema lo que pudiera ser inapropiado especialmente para países tales como Emiratos Árabes Unidos e Israel, que encuentran requerimientos de agua dulce usando desalinización de agua de mar y el uso de agua de mar desalinizada hace que no resulte en la disponibilidad reducida de agua dulce del ecosistema acuático.

\section{5) El método de Milá Canals}

El método tiene en cuenta 2 aspectos primarios a través de que el uso del agua dulce puede tener impacto en el suministro disponible:
1. Impacto del ecosistema de agua dulce (FEI).

2. Agotamiento de agua dulce [7].

El FEl es definido como el volumen de agua equivalente del ecosistema, referido al volumen de agua probable a afectar los ecosistemas. Para la valoración del FEl el método propone el uso de un indicador de estrés hídrica como un punto medio de factor de caracterización.

El indicador de estrés hídrica es definido como un rango de consumo de agua desde la cuenca de un río al agua disponible para uso humano. El agua disponible para uso humano es una diferencia entre la cantidad total de agua medio ambiental estimada, necesitada para el sostenimiento de las cuencas del ecosistema.

Las cuencas de ríos son categorizadas como las siguientes:

- Explotada ligeramente (Indicador de Estrés Hídrica $<0,7)$

- Explotada moderadamente $(0,3<\mathrm{IEH}<056)$

- Explotada pesadamente $(0,6<\mathrm{IEH}<1)$

- Sobreexplotada (lEH > 1)

A continuación, la Fig. 1 muestra el mapa de indicadores de estrés hídrica para las principales cuencas de ríos en el mundo.

Por ejemplo, muchos ríos exhiben variaciones estacionales extremas en la distribución del flujo de agua, pero el indicador de estrés hídrica que está basado en el promedio anual, no contiene tales fluctuaciones. También el método no puede ser aplicado a regiones que no pertenecen a ninguna cuenca de río, se reconoce que el uso de indicadores de estrés hídrica como el factor de caracterización pudiera ocasionar que los impactos del uso del agua de ríos se incrementen linealmente [7], por lo que los impactos del uso del agua pudieran incrementarse más rápidamente con los cambios de incrementos en los valores de estrés hídrica.

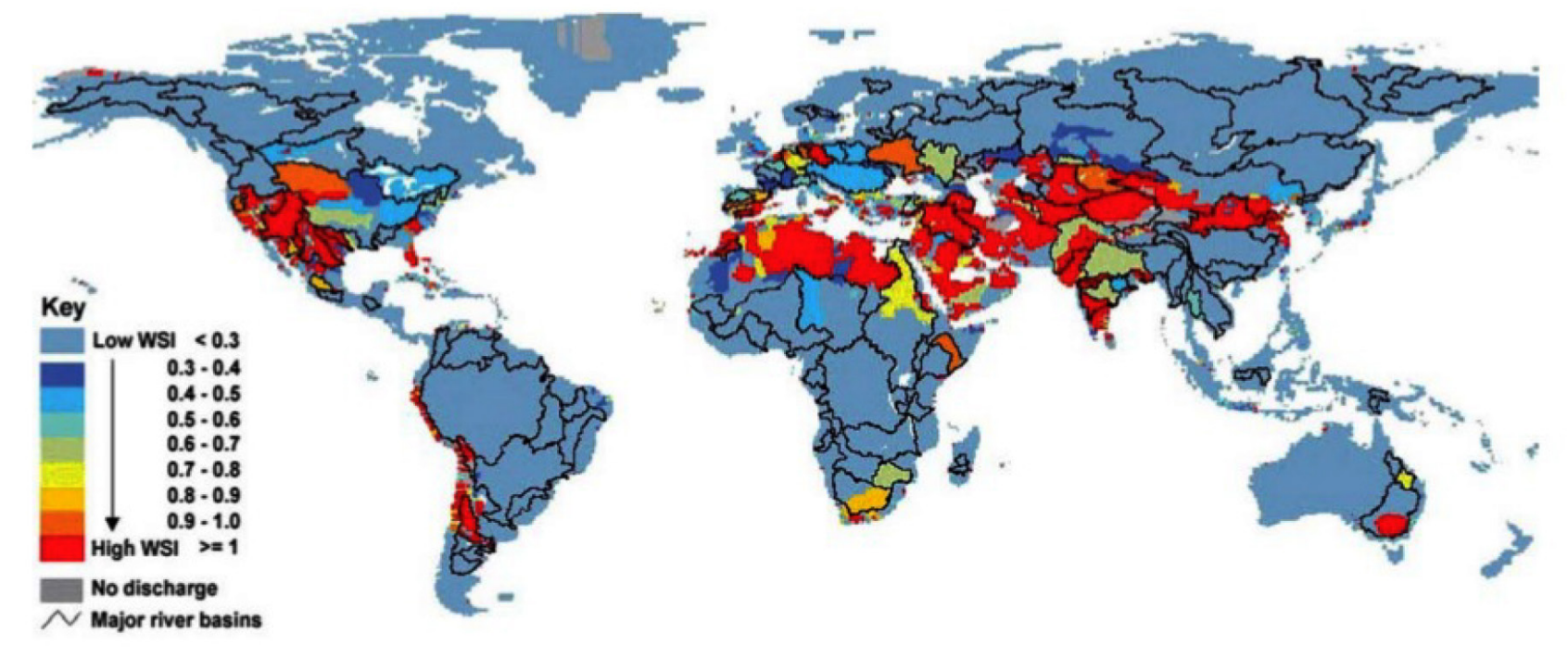

Fig. 1. Mapa de indicadores de estrés hídrica para las principales cuencas de ríos en el mundo. 


\section{6) El método de Pfister}

El método trata ambas consideraciones de factores de caracterización en el punto medio y el punto final valorando los impactos medioambientales del consumo de agua dulce [8]. Un índice de estrés hídrica (WSI) que indica los impactos del consumo de agua en relación con la escasez de agua, propuso el factor de caracterización como un punto medio. Semejante al método de eco-escasez, el índice también está basado en el WTA (Rango de escasez) y puede ser aplicado en alguna escala del espacio. También toma en consideración la variabilidad de precipitación anual y mensual como fuente, como cuenca con flujos regulados fuertemente.

A continuación, la Fig. 2 muestra los valores del WSI (Indicador de estrés hídrica), con rango desde 0,01 a 1 , los que son derivados usando la función logística siguiente:

$$
W S \mid=\frac{1}{1+g-6,4 \text { WTA } *\left(\frac{1}{0,01}-1\right)}
$$

Donde el WTA* es un WTA modificado para tener en cuenta la variabilidad de precipitación mensual y anual.

La severidad de escasez de agua de cuencas se clasifica como la siguiente:

WSI $<0,1$ Baja.

$0,1 \leq$ WSI $<0,5$ Moderada.

$0,5 \leq$ WSI $<0,9$ Severa.

WSI $>0,9$ Extrema [8], [6].

La categoría del punto final del impacto se concentra en 3 áreas relacionadas con la protección del consumo de agua: Salud humana, Calidad del ecosistema y Agotamiento de recursos de agua dulce. Estos son calculados respectivamente de acuerdo a las 3 fórmulas siguientes.

\section{- Salud humana}

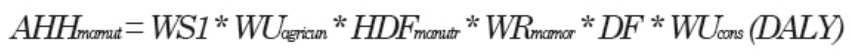

Donde:

$A H H_{\text {mamuir }}=\begin{aligned} & \text { Daño a la salud humana debido a } \\ & \text { malnutrición } \\ & \text { (en años de vida ajustados de inca- } \\ & \text { pacidad o DALY) }\end{aligned}$
$W S I=\quad \begin{aligned} & \text { Índice de estrés hídrico. } \\ & W U_{\text {agricon }}= \\ & H D F_{\text {mamuir }}=\begin{array}{l}\text { Fracción de uso de agua en la agricul- } \\ \text { tura en el nivel de la cuenca. }\end{array} \\ & \text { Factor de desarrollo humano, vincu- } \\ & \text { lando el índice de desarrollo humano } \\ & \text { a la vulnerabilidad de malnutrición. }\end{aligned}$
$D F=\quad \begin{aligned} & \text { Requerimiento de agua para preve- } \\ & \text { nir la malnutrición (m² personas año) } \\ & \text { Daños debido a malnutrición (DALY/ } \\ & \text { pers. año) } \\ & \text { Consumo de agua azul (m”) }\end{aligned}$

- Calidad del ecosistema

$\Delta E Q=\frac{N p p_{\text {wat }}-\lim }{p} *$ WUcons. $\left(\mathrm{m}^{2} \cdot \mathrm{añ} 0\right)$

Donde:

$A E Q=$ Daño a la calidad del ecosistema $\left(\mathrm{m}^{2} *\right.$ año)

$N P P=$ Limitada producción primaria neta por vulnerabilidad y déficit de agua.

$P \quad=\quad$ Lluvia anual media (m/año)

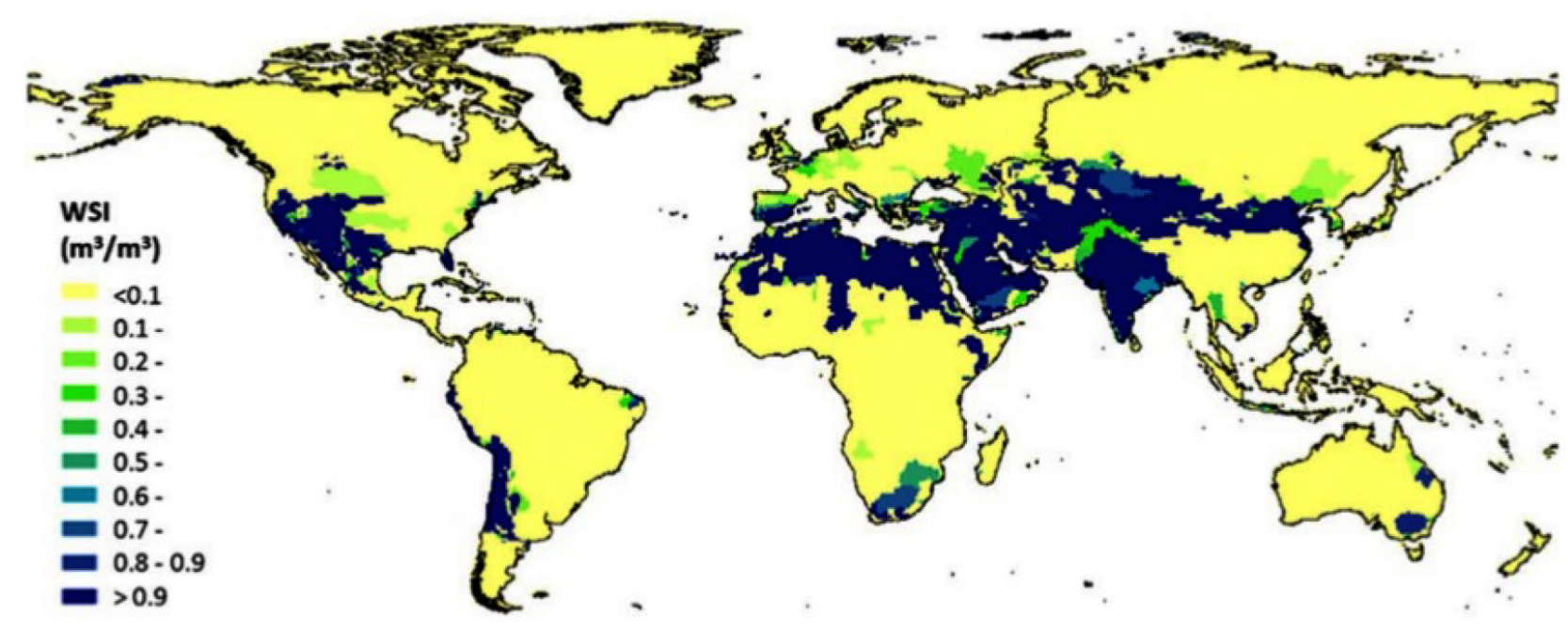

Fig. 2. Mapa de estrés hídrica en el nivel de cuenca. 
- $\quad$ Agotamiento de recursos de agua dulce

$\Delta R=E_{\text {desalin. }} \times F_{\text {agotam }} \times W U_{\text {cons }}$.

Donde:

$A R=$ Daño a los recursos de agua dulce $(\mathrm{MJ})$

$E_{\text {desatin }}=$ Energía requerida para la desalinización de agua de mar $\left(\mathrm{MJ} / \mathrm{m}^{3}\right)$

$F_{\text {agotam }}=$ Fracción del consumo de agua dulce que contribuye al agotamiento del recurso.

$W U_{\text {cons }}=$ Consumo de agua azul $\left(\mathrm{m}^{3}\right)$

Las categorías de impacto relacionadas en el método están en conjunto dentro de puntuación simple como por el método del Procesador-Eco 99. El impacto debido al consumo de agua es expresado como E199HA puntos $/ \mathrm{m}^{3}$.

Considerando que estos cálculos están ideados para convenir los datos disponibles, ellos representan un intento para desarrollar un indicador que refleje los impactos locales, a su vez el método no presenta ningún modelo de impactos ecológicos del agua residual.

A continuación, se ofrecen los resultados de un estudio en la producción de etanol (Tabla 1).

\section{Valoración de los métodos}

La evaluación de la huella hídrica según los métodos abordados demuestra un buen nivel científico, han sido validados con estudios en diferentes regiones del mundo, se han aplicado en la valoración de los mapelos del uso del agua en el proceso de producción de bioetanol a partir del maíz, en el cálculo de la huella hídrica de productos agrícolas, cárnicos, en hidroeléctricas, procesos industriales, etc, con buenos resultados, se observan en general las insuficiencias siguientes:

- Información limitada del contenido de las bases de datos para la valoración del ciclo de vida siendo simple el reporte del total de agua en uso.

- La mayoría de las bases de datos no consideran siempre la cantidad de agua residual y no reportan el volumen de agua utilizado en la producción.

- La distinción entre la entrada y salida del flujo de agua es esencial para una adecuada valoración de los impactos potenciales, lo cual no se relaciona por lo general.

- Hay inadecuaciones del nivel de inventario de datos y la valoración del ciclo de vida y generalmente no se considera conveniente para la cuantificación del uso del agua.

Se aprecia en la estructuración de los métodos falta de integralidad y de enfoque multicriterio al no considerar en muchos casos todas las aguas utilizadas en las industrias como el agua gris o residual, el consumo de agua en limpiezas, mantenimientos, cocción de alimentos y otros servicios, tampoco se valoran los impactos asociados a factores como son la eficiencia energética, aspectos técnico-económicos de explotación eficiente de equipos, los consumos energéticos y los costos, la influencia de la calidad del agua azulo de consumo, cuyos factores sino se tienen en cuenta pueden incrementar los costos de producción, los consumos de agua y el estrés hídrica, en detrimento del uso racional y eficiente del agua y del ahorro de energía [10].

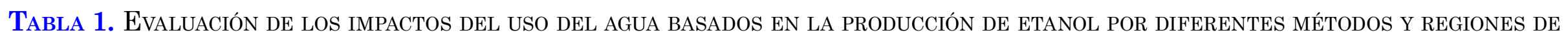
PAÍSES.

\begin{tabular}{|c|c|c|c|c|c|c|c|c|c|c|}
\hline \multirow[t]{2}{*}{ Región } & \multirow[t]{2}{*}{$\begin{array}{c}\text { Total } \\
\text { Agua Azul } \\
\text { consumida }\end{array}$} & \multicolumn{2}{|c|}{$\begin{array}{l}\text { Método de Eco-escasez } \\
\text { (nivel de país) }\end{array}$} & \multicolumn{3}{|c|}{$\begin{array}{l}\text { Método (cuenca del río) } \\
\text { (Milá) }\end{array}$} & \multicolumn{2}{|c|}{$\begin{array}{c}\text { Método (WSI - Índice de } \\
\text { estrés hídrico de cuenca) } \\
\text { (Pfister) }\end{array}$} & \multicolumn{2}{|c|}{$\begin{array}{l}\text { Método (Impactos del } \\
\text { consumo de agua en } \\
\text { cuenca) (Pfister) }\end{array}$} \\
\hline & & $\begin{array}{l}\text { Factores de } \\
\text { caracterización } \\
\left(\mathrm{EP} / \mathrm{m}^{3}\right)\end{array}$ & $\begin{array}{l}\text { Impactos } \\
\text { del uso } \\
\text { del agua } \\
\text { (EP/GJ)a }\end{array}$ & $\begin{array}{l}\text { Efectos } \\
\text { del uso } \\
\text { de la } \\
\text { tierra } \\
\text { en el } \\
\text { agua } \\
\left(\mathrm{m}^{3} / \mathrm{GJ}\right)\end{array}$ & $\begin{array}{l}\text { Factores de } \\
\text { caracterización }\end{array}$ & $\begin{array}{l}\text { Impactos } \\
\text { del uso } \\
\text { del agua } \\
\text { (Ecosist- } \\
\text { eq.Agua) } \\
\left(\mathrm{m}^{3} / \mathrm{GJ}\right) \mathrm{b}\end{array}$ & $\begin{array}{l}\text { Factor de } \\
\text { caracterización } \\
\left(\mathrm{EP} / \mathrm{m}^{3}\right)\end{array}$ & $\begin{array}{l}\text { Impactos } \\
\text { del uso } \\
\text { del agua } \\
\left(\mathrm{m}^{3} / \mathrm{GJ}\right) \mathrm{c}\end{array}$ & $\begin{array}{l}\text { Factor de } \\
\text { caracterización } \\
{[8]}\end{array}$ & $\begin{array}{l}\text { Impactos } \\
\text { consumo } \\
\text { del agua } \\
\text { (puntos/ } \\
\text { GJ)c }\end{array}$ \\
\hline $\begin{array}{l}\text { Ilinois } \\
\text { (USA) }\end{array}$ & 26,45 & 220 & 5820 & 1,9 & 0,69 & 19,65 & 0,01 & 0,29 & 0,02 & 0,44 \\
\hline $\begin{array}{l}\text { Jilin } \\
\text { (China) }\end{array}$ & 17,03 & 880 & 14,98 & 6,1 & 0,05 & 1,16 & 0,12 & 2,12 & 0,02 & 0,05 \\
\hline $\begin{array}{l}\text { Parana } \\
\text { (Brazil) }\end{array}$ & 9,51 & 24 & 22,8 & 5,9 & 0,03 & 0,52 & 0,01 & 0,11 & 0,01 & 0,05 \\
\hline
\end{tabular}

a. Impactos del consumo de agua = 1/4 Total de agua extraída * Factor de caracterización. b. Impactos del consumo de agua = 1/4 Total de agua consumida + efectos del uso de la tierra en el agua * Factor de caracterización. c. Impactos del consumo de agua = 1/4 Total de agua consumida * Factor de caracterización. 


\section{Conclusiones}

1. Los métodos, metodologías e indicadores para la valoración de los impactos del uso del agua dulce están estancados. El concepto de huella hídrica ha sido un importante paso en esta dirección. Sin embargo, la huella hídrica volumétrica no dirige la cuestión de disponibilidad de agua o escasez que es frecuente, lo que concierne en los impactos del consumo de agua dulce.

2. Se han discutido los indicadores de estrés hídrica en las naciones o las inevitables grandes variaciones en los niveles de cuencas de ríos, especialmente para países diversos geográficamente y con grandes ríos. Los factores de caracterización deben ser basados sobre la información específica situada sobre la disponibilidad de agua y su uso, cuya disponibilidad de tal tipo de datos es un problema en muchos países.

3. Los factores de caracterización del nivel de cuencas proporcionan una alternativa útil para el estudio de caso del etanol del maíz, revelando que estos factores también tienen algunas limitaciones.

4. Los indicadores de nivel de cuencas de ríos, están basados en los datos anuales y no pueden considerar las variaciones estacionales con respecto a la disponibilidad de agua. Además, los impactos medioambientales del consumo de agua no sólo dependen de la escasez de agua, también de la magnitud y frecuencia de impactos de diluvios y sequías, resiliencia y vulnerabilidad de los ecosistemas.

5. se demuestra que el daño a los ecosistemas debido al agua sólo es considerado en uno de los métodos (el Pfister), mientras otros métodos no tienen en cuenta estos parámetros, por lo que se hacen necesarios desarrollos metodológicos en los métodos de evaluación para mantener una base de parámetros más fuerte que resuelva estos problemas.

6. la utilización de datos de calidad en el uso de agua para varios propósitos es un factor que está limitando para valorar los impactos del uso del agua. Para muchos procesos industriales y agrícolas, los datos del uso del agua actuales son escasos porque pocas compañías y agricultores coleccionan o reportan el uso del agua.

7. Situaciones como la falta de mediciones del uso del agua subterránea y de la descarga de agua residual hace difícil estimar correctamente el consumo de agua.

8. Es muy importante crear una metodología estandarizada integral para la valoración de los impactos del uso del agua sobre bases del ciclo de vida que considere todas las aguas del proceso evaluado y de otros usos, así como otros impactos energéticos, técnico-económicos y de la calidad del agua en función del uso eficiente del agua y el ahorro de energía.
9. La investigación en el tema de la huella hídrica debe analizar si la valoración del ciclo de vida es una herramienta apropiada para dirigir estos problemas $\mathrm{u}$ otras herramientas deben ser empleadas.

\section{REFERENCIAS}

[1] J. A. Allan, "Virtual water: a strategic resource, global solutions to regional deficits", Groundwater, vol. 36, no. 4, pp. 545-546, Dec. 2005. https://doi. org/10.1111/j.1745-6584.1998.tb02825.x

[2] A. Y. Hoekstra y A. K. Chapagain, Globalization of Water: Sharing the Planet's Freshwater Resources. Oxford, UK: Blackwell Publishing Ltd., 2008. https://doi. org/10.1002/9780470696224

[3] A. Y. Hoekstra, A. K. Chapagain, M. M. Alday \& M. Mekonnen, Mater footprint manual: State of the Art 2009, Enschede, NL: Water Footprint Network, 2009. Available from https://waterfootprint.org/media/downloads/WaterFootprintManual2009.pdf

[4] B. G. Ridoutt, S. J. Eady, J. Sellahewa, L. Simons \& R. Bektash, Walter footprinting at the product brand level: case study and future challenges," J Clean Prod, vol. 17, no. 13, 1228-1235, Sept. 2009. https://doi.org/10.1016/j. jclepro.2009.03.002

[5] W. W. F. Miller, Water Footprinting: Identifying \& Addressing Water Risks in the Value Chain, Woldaming, UK: WWF/SAB Miller, 2009.

[6] B. G. Ridoutt \& S. Pfister, "A revised approach to water footprinting to make transparen the impacts of consumption and production on global freshwater searcity", Global environ chang, vol. 20, no. 1, 113-120, Feb. 2010. https://doi.org/10.1016/j.gloenvcha.2009.08.003

[7] L. M. Canals, J. Chenoweth, A. Chapagain, S. Orr, A. Anton \& R. Clift, "Assessing freshwater use impacts in LCA: partl e inventory m odelling and characterisation factors for the main impact pathways," Int J Life Cycle Ass, vol. 14, no. 1, pp. 28-42, Jan. 2009. https://doi.org/10.1007/ s11367-008-0030-z

[8] S. Pfister, A. Koehler \& S. Hellweg, "Assessing the environmental im pacts of freshwater consumption in LCA," Environ Sci Technol, vol. 43, no. 11, pp. 4098-4104, Apr. 2009. https://doi.org/10.1021/es802423e

[9] M. Bertolli, G. Roark, S. Urrutia y F. Chiodi, "Revisión de modelos de madurez en la medición del desempeño", INGE CUC, vol. 13, no. 1, pp. 70-83, 2017. https://doi. org/10.17981/ingecuc.13.1.2017.07

[10] Y. De la Peña, G. Bordeth; H. Campo \& U. Murillo "Clean Energies: An Opportunity to Save the Planet", IJMSOR, vol. 3, no. 1, pp. 21-25, 2018. https://doi.org/10.17981/ijmsor.03.01.04 